\title{
INTERPRETATION OF RADIO ECHOES FROM STORGLACIÄREN, NORTHERN SWEDEN
}

\author{
By M. E. R. WALFORD, M. I. KENNETT, \\ (H. H. Wills Physics Laboratory, University of Bristol, Bristol BS8 1TL, England) \\ and $\mathrm{P}$. HOLMLUND
}

(Naturgeografiska Institutionen, Universitet Stockholms, Drottninggaten 120, Stockholm, Sweden)

\begin{abstract}
Storglaciăren (lat. $67.5^{\circ} \mathrm{N}$., long. $17.5^{\circ} \mathrm{E}$.) is a sub-polar glacier which has been the object of detailed study for many years. It responds in a sensitive way to annual and long-term changes in climate but it does not surge. Conditions at the bed and the distribution of englacial water are of considerable interest. In this paper we discuss an attempt to learn something about these matters by radio-echo soundings at metric and decametric wavelengths. We analyse radio-echo records mainly using a scalar-wave theory of the diffraction of pulses. The pulse shapes of echoes are useful because they help us to recognize the types of target and processes responsible for the echoes. We then use simple statistical measurements of radio echoes to provide estimates of certain average properties of the targets. We estimate, for example, the roughness of the glacier bed and the distribution and orientations of scatterers within the ice.
\end{abstract}

RÉsumé. Interprétation des échos radio, Storglaciären, Suède septentrionale. Le Storglaciären $\left(67,5^{\circ} \mathrm{N} ; 17,5^{\circ} \mathrm{E}\right)$, glacier de type sub-polaire, est bien étudié depuis de nombreuses années. Bien que répondant de manière très sensible aux fluctuations climatiques, il ne surge pas. Les conditions régnant contre le lit et la répartition de l'eau présentent un intérêt considérable. Dans cet article nous examinons une tentative d'étudier ces caractéristiques par des sondages radio utilisant des longueurs d'ondes métriques et décamétriques. Nous analysons les enregistrements des échos radio principalement à l'aide d'une théorie d'onde scalaire

\section{LIST OF SYMBOLS}

$\vec{a}$

$A$

$\vec{b}$

B

$c$

6

D

$\vec{E}$

$\vec{E}$

E

$\epsilon_{\mathrm{i}}$

$\epsilon_{0}$

$f_{\mathbf{v}}$
A vector $\vec{a}=\vec{n}_{\wedge} \vec{z}_{\wedge} \vec{n}$

Area of receiving antenna

An angular measure of the spread of wave vectors

Wave speed in a medium, usually ice

Coherency parameter

Thickness of water film

Electric field

Energy in a transmitted pulse

Relative permittivity of medium i

Permittivity of free space

Volume fraction occupied by water in ice

Surface fraction of bed covered by water
A unit vector parallel to an antenna axis

pour la diffraction des impulsions. La forme des impulsions d'écho sont utiles pour l'aide à la reconnaissance des types de cibles et des processus responsables de ces échos. C'est ainsi qu'une statistique simple sur les mesures des échos nous fournit des estimations de certaines propriétés des réflecteurs. Nous estimons, par exemple la rugosité du lit glaciaire et la distribution et les orientations des diffuseurs à l'intérieur de la glace.

ZUSAMMENFASSUNG. Interpretation von Radio-Echolotungen am Storglaciären, Nord-Schweden. Der Storglaciären (67.5 nördl.Br., $17.5^{\circ}$ ostl.L.) ist ein subpolarer Gletscher, der durch viele Jahre eingehend untersucht worden ist. $\mathrm{Er}$ reagiert empfindlich auf jăhrliche und langfristige Klimaschwankungen, zeigt jedoch keine Ausbrüche. Von besonderem Interesse sind die Verhăltnisse am Felsbett und die Verteilung von Wasser im Eiskörper. Der folgende Beitrag beschreibt einen Versuch, mit Hilfe von RadioEcholotungen bei Wellenlăngen im Meter- und DekameterBereich einige dieser Fragen aufzuklären. Unsere Analyse der Radio-Echoaufzeichnungen stützt sich meist auf eine skalare Wellentheorie der Impulsdiffraktion. Die Impulsformen von Echos sind von Bedeutung, weil sie zur Erkennung der Reflexionsstellen und der echo-erzeugenden Prozesse beitragen. Es folgt dann eine einfache statistische Auswertung von Radio-Echos, die Abschätzungen für bestimmte mittlere Eigenschaften der Reflexionsstellen liefert. Zum Beispiel lässt sich so die Rauhigkeit des Gletscherbettes sowie die Verteilung und Ausrichtung von Streukorpern im Eis ermitteln.

$F(t)$

$\phi$

$g(r)$

G

$h$

$L$

$\lambda$

n

$\vec{n}$

$N$

$P_{\mathrm{T}}(t)$

$P(t)$

$P_{i j}(t)$

\section{Wave form of transmitted pulse}

A phase

Effective target strength as a function of range

Antenna gain

Glacier depth

Horizontal measure of surface irregularities

Wavelength or central wavelength of a pulse in ice

A number

A vector Number of englacial scatterers per unit
volume

Transmitted pulse power

Radio-echo power

Radio-echo power in a polarization experiment 
$\psi(t)$

$\vec{r}$

$r$

$R_{1}, R_{2}$

$S$

$\sigma$

$t, t^{\prime}$

$T$

$\tau$

$\theta_{0}$
Radio-echo scalar wave

A range vector to a distant point

$|\vec{r}|$

Principal radii of curvature of a surface

Vertical measure of surface irregularities

Radio-echo scattering cross-section of an independent target

Measures of time

Echo half-length

Wave absorption characteristic time at $65 \mathrm{MHz}$

Azimuthal angle w.r.t. a fixed bearing

\section{INTRODUCTION}

In this paper we discuss the interpretation of recent results from a continuing programme of radio-echo sounding at Storglaciăren (Fig. 1) in northern Sweden. First we describe the echo sounders. This is followed by two main sections of the paper: one deals with radio echoes returned from the bed of the glacier and the other with echoes from englacial targets.

Echoes from the glacier bed are clearly recognizable on records obtained with low-frequency, monocycle, echo sounders. Björnsson (1981) has mapped the ice thickness using such records. We are interested in what more can be learnt by radio-echo sounding. The pulse shape of the echoes depends on the geometrical shape and roughness of

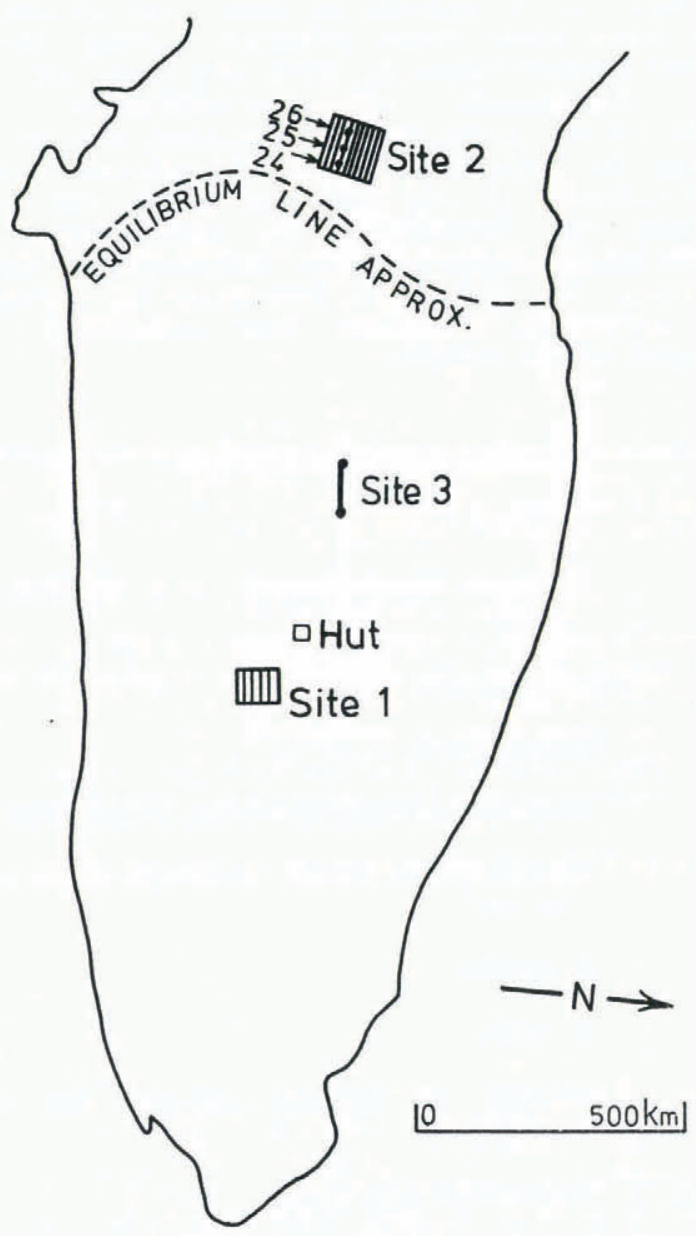

Fig. 1. Sketch map of Storglaciarren showing the principal sites referred to in this paper.

\section{$U_{\mathrm{R}}(t), U_{\mathrm{T}}(t) \quad$ Impulse response functions}

$V(t) \quad$ Dimensionless function proportional to antenna driving voltage

$v(t) \quad$ Voltage signal at the terminals of a receiving antenna

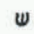

Angular frequency of a wave or central angular frequency of a pulse

$x \quad$ Coordinate measure

$Z, Z_{1}, Z_{\mathrm{iw}}, Z_{\mathrm{wr}}$ Amplitude coefficient of reflection

$z \quad$ Coordinate measure

$\vec{z} \quad$ Unit vector parallel to antenna the bed and also on physical conditions, for example, on the possible presence of water. By analysing the pulse shapes of echoes, therefore, we are able to place some limit on the probable roughness and curvature of the bed, and on the amount and distribution of water.

Echoes returned from englacial targets at Storglaciären are much stronger than echoes returned from within polar ice masses and they exhibit no stratifications. We find that our radio-echo observations can be accounted for in terms of a simple model of scattering by targets distributed at random through the glacier below a depth of $30-40 \mathrm{~m}$. A computer program based on such a model has been used to generate simulated radio-echo records which bear a strong resemblance to the field records. We can estimate the mean back-scattering cross-section per unit volume of ice from the field data.

Echo-polarization measurements suggest, however, that the targets are substantially anisotropic in shape with a tendency to be aligned with respect to the direction of the ice flow.

\section{RADIO-ECHO SOUNDING EQUIPMENT}

We have used two low-frequency monocycle echosounders and one $65 \mathrm{MHz}$ phase-sensitive echo-sounder. One of the monocycle instruments, which we refer to as model $\mathrm{B}$, was built in Bristol. It has two resistively loaded dipole antennae. The transmitting antenna is coupled to the transmitter by a balancing transformer and is excited by the discharge of a capacitor through a fast-switching thyristor, type XT-2104. This instrument transmits a pulse which has an approximately monocycle wave form with a central wavelength in ice, $\lambda$, of $25-30 \mathrm{~m}$ (Fig. 2a). The receiving antenna is physically identical to the transmitting antenna. For the experiments described in this paper, it was coupled to a portable oscilloscope by means of a simple impedencematching pad which was adjusted in the field for optimum performance. A balanced-to-unbalanced transformer did not appear to improve the performance. The radio echoes were recorded in A-scope format by photographing the oscilloscope display. This system worked well, apart from occasional triggering instabilities. The receiving and transmitting equipment was easily carried by two men.

The second low-frequency monocycle echo-sounder is designated as model $\mathbf{S}$ and is owned by the University of Stockholm. The electronic design and performance is very similar to model B but it is usually operated with the transmitter and receiver mounted on sledges which are towed behind a snow vehicle so that the two resistively loaded antennae travel in line astern. The echoes are recorded on film by photographing a monitor display, usually in I-scope mode, but sometimes in A-scope mode.

The third echo-sounder is a $65 \mathrm{MHz}$ quasimonochromatic phase-sensitive instrument. It is bistatic and uses two resonant antennae. It transmits a pulse containing approximately 10 cycles of the carrier wave. Radio 

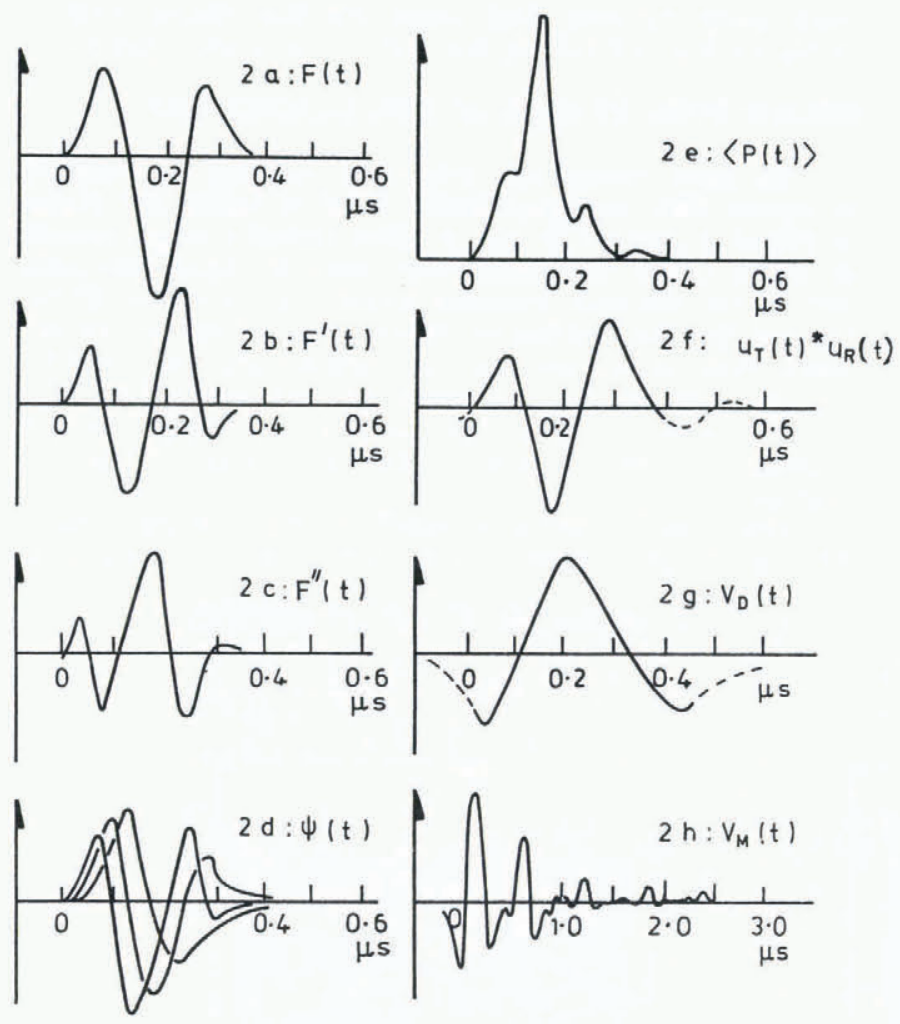

Fig. 2. Various pulse shapes. (a) $F(t)$ : the radio signal transmitted by system $B$, observed with the receiving antenna $20 \mathrm{~m}$ to one side of the transmitting antenna and parallel to it. (b) $F^{\prime}(t)$ : the first derivative of $F(t)$ representing the echo to be expected from a water layer $0.1 \mathrm{~m}$ thick. (c) $F^{\prime \prime}(t)$ : the second derivative of $F(t)$ representing the echo to be expected from a target much smaller than $\lambda .(d)$ $\Psi(t)$ : three wave forms illustrating the range of pulse shapes commonly found among echoes returning from the glacier bed. $(e)\langle P(t)\rangle$ : mean echo power as a function of delay time calculated from A-scope records. ( $f$ ) $U_{\mathrm{T}}(t) * U_{\mathbf{R}}(t) * V(t):$ a version of $F(t)$ calculated from fundamental wave theory. $(\mathrm{g}) V_{\mathrm{D}}(t)$ : the directly coupled signal between antennae approximately $\lambda / 2$ apart. ( $h$ ) $V_{\mathrm{M}}(t)$ : multiple echoes returned from bore hole 83.12 (Fig. $3 a)$. Note the change of time-scale.

echoes are recorded by photographing an oscilloscope display of the received signals.

Now we shall discuss the behaviour of the resistively loaded dipole antennae. When a voltage is applied to the antenna terminals, voltage and current waves propagate along the wires and are smoothly attenuated so that negligible energy is reflected from the ends of the wires (Wu and King, 1965). Kanda (1980) has computed and measured the impulse response, of such an antenna in transmission, $U_{\mathrm{T}}(t)$, and in reception, $U_{\mathrm{R}}(t)$. If we apply an impulsive voltage signal to an antenna, the electric field produced at a far field point, $\vec{r}$, is given by the expression

$$
\vec{E}_{\delta}(\overrightarrow{r, t})=\frac{\vec{a}}{r} U_{\mathrm{T}}(t-r / c)
$$

where $r=|\vec{r}|, \vec{a}=\vec{n}_{\wedge} \vec{z} \wedge \vec{n}, \vec{n}=\vec{r} / r$, and $\vec{z}$ is the unit vector parallel to the antenna. $c$ is the wave speed in the surrounding medium. If, more generally, $V(t)$ is a dimensionless function of time proportional to the voltage signal applied to the terminals, then the far field is given by the convolution integral

$$
\vec{E}(\vec{r}, t)=\int_{-\infty}^{+\infty} \vec{E}_{\delta}\left(\vec{r}, t^{\prime}\right) V\left(t-t^{\prime}\right) \mathrm{d} t^{\prime} .
$$

Let the receiving antenna be placed at $\vec{r}$ and aligned to some unit vector $\vec{b}$. The voltage signal appearing at the output terminals of this antenna is:

$$
V(t)=\int_{-\infty}^{\infty} \vec{b} \cdot \vec{E}\left(\vec{r}, t^{\prime}\right) U_{\mathbf{R}}\left(t-t^{\prime}\right) \mathrm{d} t^{\prime} .
$$

Kanda has pointed out that, for a given antenna, $U_{\mathrm{T}}(t)$ is equal to the time derivative of $U_{\mathrm{R}}(t)$. We have scaled Kanda's results so that they may be compared with our monocycle radio-echo signals. We convolved the functions $U_{\mathrm{R}}(t)$ and $U_{\mathrm{T}}(t)$ together and then convolved the result with a suitable voltage pulse which models the pulse applied to our transmitting antennae. Thus, we have calculated the signal voltage $V(t)$ which would be produced at the receiver terminals by a plane mirror. The result is shown in Figure $2 \mathrm{f}$. Compare this with Figure $2 \mathrm{a}$, which shows the signal obtained in the field with the transmitting and receiving antennae approximately parallel to each other. The differences between Figure $2 a$ and $f$ are mainly due to the different resistive profiles used in Kanda's calculations and our experimental practice.

Transmission, propagation, scattering, and reception are processes which may be represented mathematically as linear processes of convolution. Such linear processes can be performed in any order without altering the final result. Because of this, we may make the following useful conceptual simplification: we may imagine that, during echosounding, the processes of transmission and reception are performed first and produce the scalar pulse $V(t)$ shown in Figure 2a. This pulse we refer to as the elementary wavelet. We now imagine that it is applied to the terminals of an ideal transmitting device for which $U_{\mathrm{T}}(t)=\delta(t)$, where $\delta(t)$ is the impulse function of unit strength. After propagation and reflection, the echo is received using an ideal receiver for which $U_{\mathrm{R}}(t)=\delta(t)$. For many purposes it is helpful to think in this was as if $V(t)$ actually represented the pulse shape of the transmitted wave and the antennae introduced no distortion.

For convenience, when we traverse using echo-sounder $S$, we usually operate with the two antennae lying with their long axes in the same straight line, separated by approximately one antenna length. In this configuration, the signal directly radiated from the transmitting to the receiving antenna should, by symmetry, be zero. More accurately, if we take account of the presence of the glacier surface, which breaks the axial symmetry of the antenna configuration, we should expect the coupling between the antennae to be small and very sensitive to the precise antenna orientation. In practice, the initial signal appearing at the receiving antenna is strong, very insensitive to the precise antenna orientation, and varies negligibly as the antenna system is moved along the line of a traverse. Even more surprisingly, the wave form, which is shown in Figure $2 \mathrm{~g}$, is quite unlike the signal shown in Figure 2a: its pulse length, for example, is approximately twice as long. It is important to understand why this is so, because in the field this direct signal is used to trigger the oscilloscope display and to provide a working origin of time when we measure radio-echo delay times.

We believe that the direct signal arises from a retarded dipole-dipole interaction which is essentially inductive in nature, rather than radiative. Electrostatic induction is particularly likely to be important for resistively loaded antennae because of the relatively high voltages which must be used to drive current through the resistors. When the antennae are about half a wavelength apart, the induced signal consists of three half-cycles of alternating polarity, as illustrated in Figure $2 \mathrm{~g}$. This we would expect in view of the symmetry of the dipole-dipole arrangement, remembering that the excitation is provided by a brief voltage pulse of single polarity. The duration of the induced signal, $0.4 \mu \mathrm{s}$, is longer than that of the radio echo, perhaps because of the different travel times that signals coupled between different parts of the antennae system take to arrive at the receiver terminals. Also likely to be important are the matching conditions at the antennae terminals and, possibly, the distribution of ice and water near the antennae. With the antennae very close together, the direct 
signal is dominated by a single positive voltage excursion. Field measurements over the small range of separations for which this condition holds show that the coupled signal varies with separation according to an inverse-square law. Thus the coupling approximates a pole-pole interaction between the neighbouring halves of the two antennae. There is an effective pole centre for this interaction which we find lies about half-way along each antenna pole. These results lend some support to the hypothesis of electrostatic induction.

The details of the antenna coupling are peculiarly difficult to calculate. Even if we knew the inductive electric field produced by the transmitting antenna, it would be very difficult to calculate the voltage signal induced at the receiver terminals, for this would depend in a complicated fashion on the resistive-loading profile and on the matching impedance. We must therefore proceed experimentally to establish the correct origin of time to be used when measuring echo-delay times. We shall discuss experimental methods of solving this problem in a later section.

\section{PULSED ECHO STUDIES OF THE GLACIER BED}

We have carried out radio-echo sounding experiments at various sites on Storglaciăren, some of which are shown in Figure 1. Figure 3a and b show typical I-scope records in which $t$, the radio-echo delay time is plotted vertically and $x$, the distance traversed across the snow, is plotted horizontally. The relationship between the voltage at the antenna terminals of the receiving antenna and the intensity on film is monotonic, non-linear, and not well known, and so we augment the I-scope record by taking frequent A-scope pictures which show the wave form at selected positions.

Our records illustrate a wealth of different phenomena. We may recognize echoes which seem to be reflections from
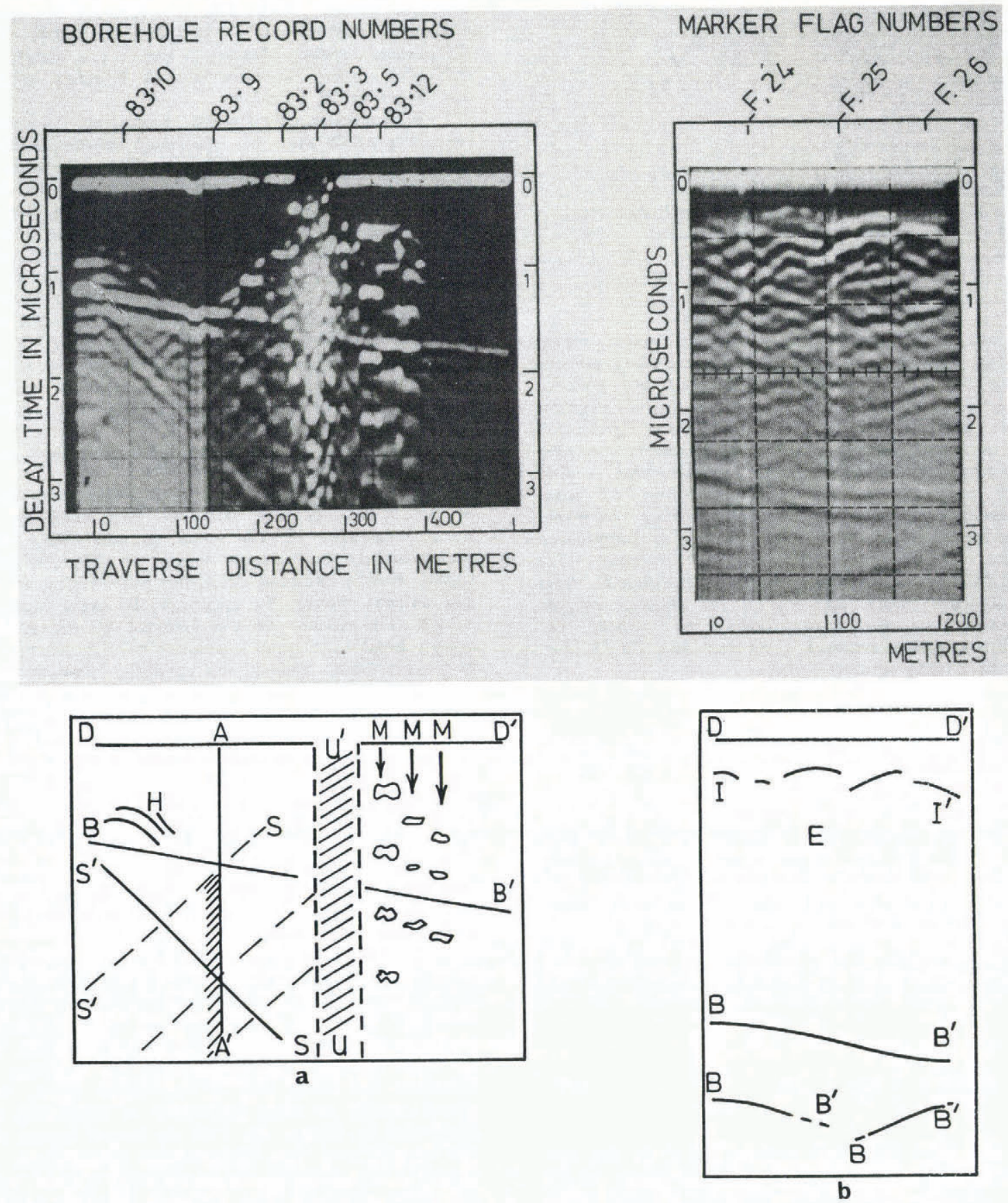

Fig. 3. I-scope recordings from Storglaciären, system S. Radio-echo delay time is plotted vertically and vehicle position horizontally. (a) Example showing the direct signal DD', the echo from the bed $\mathrm{BB}^{\prime}$, hyperbolic echo trajectories $\mathrm{H}$, straight echo trajectories $\mathbf{S S}$ ', and multiple echoes $\mathrm{M}$. Poor synchronization probably caused the effect seen at $\mathrm{UU}^{\prime} . \mathrm{AA}^{\prime}$ is an artifact of the photographic recording. (b) Example showing several echoes from the bed $\mathrm{BB}$ ' and echoes from englacial targets $\mathbf{E}$. 
the glacier bed, others which arise from englacial targets such as water bodies, and yet others which seem to be connected with artifacts. In general, one would need to use a vector-wave theory of scattering in order to analyse such complicated scattering effects, and to allow for the polarization properties of the antennae. However, we may legitimately make use of an approximate, scalar-wave theory, in order to analyse, for example, the continuous echo BB' $^{\prime}$, in Figure 3a, which is produced by reflection from the glacier bed. It is appropriate to use a scalar theory of diffraction to describe the echoes returned from the surface, if it is substantially free from cracks, cavities, boulders, or edges sharp on a wavelength scale. Such surfaces do not significantly alter the polarization state of linearly polarized radio waves which are reflected at near normal incidence. We shall use a scalar-diffraction theory of the scattering of pulses from rough surfaces, which was developed on the basis of Kirchhoff theory by Berry (1972, 1973). Berry assumed a paraxial, far-field geometry and supposed that the reflecting wavy surface is freed from shadowing effects and multiple reflections.

Following Berry, we may express the echo in the form of a convolution integral thus:

$$
\psi(t)=-\frac{1}{2 \pi c} \int_{0}^{\alpha} F^{\prime}(t-2 r / c) g(r) \mathrm{d} r .
$$

$F(t)$ corresponds to the elementary wavelet $V(t)$ discussed in connection with Equation (3). The ' denotes differentation with respect to the argument and $c$ is the speed of radio waves in the medium. The distance from the approximately coincident source-receiver to a point on the reflecting surface is $r$ and $g(r)$ is a geometrical function which represents the effective reflection coefficient of the target as a function of range. It takes the inclination factor of the surface properly into account. Berry gave various useful expressions for $g(r)$. For example, he showed how it can be written as an integral around contours of constant range in the reflecting surface. He pointed out that, if the reflection coefficient $z$ of the surface is uniform over the target, then the echo is dominated by contributions from near the geometrical reflecting points of the surface: these points are associated with singularities in the function $g(r)$.

On the record shown in Figure 3a, which was obtained with the low-frequency echo-sounder, model $\mathrm{S}$, the echo

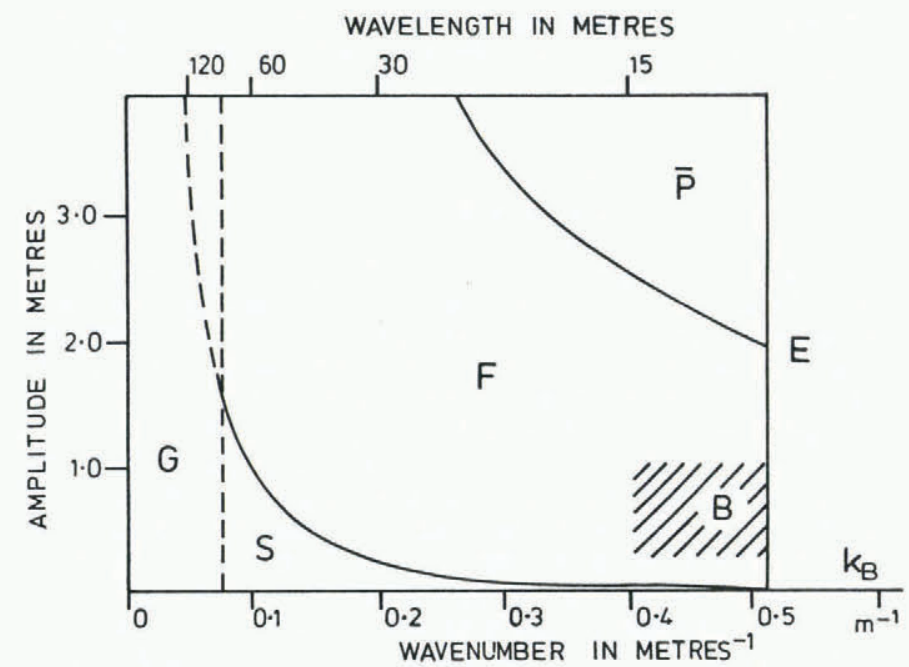

Fig. 4. Mapping of the bedrock charcteristics which affect radio echoes. The ordinate gives the amplitude of $a$ sinusoidal wavy component of bedrock roughness, the wave number is plotted as abscissa. Regions are indicated as follows: G: geographical scale undulations; E: short-wavelength region reflecting evanescent waves; $\mathrm{P}$ : non-paraxially reflecting region; $\mathbf{F}$ : region bounded by line $\mathrm{L}$ within which focussing of echoes is produced; s: region reflecting specular echoes; B: bedrock roughness of Storglaciären measured from radio-echo statistics. from the glacier bed is represented by $\mathrm{BB}^{\prime}$ which is, to a good approximation, a smooth and continuous band. These qualities are very significant because they place considerable constraint on models of the glacier bed. To start with, they suggest that focussing of the echoes by the glacier bed does not occur in this region, at least not on the larger, geographical scale sizes, corresponding to the glacier thickness. In a strict geometrical sense, a bed that produces no focussing has both its principal radii of curvature everywhere greater than the local ice thickness. In Figure 4 we have plotted a line $\mathrm{L}$ which shows the maximum amplitude of a sinusoidal corrugation of the bedrock surface which does not produce focussing of echoes. A corrugation of wavelength $60 \mathrm{~m}$ cannot exceed $1 \mathrm{~m}$ in amplitude without producing visible focussing effects in ice more than $100 \mathrm{~m}$ deep. Some focussing effects are exemplified in Figure $3 \mathrm{~b}$ : this record, from a deep narrow part of the glacier, shows more than one smooth, identifiable bedrock echo. Each echo is returned from a different area of the bed and varies in range as the instrument travels along. We would expect to be able to find a region where two such echoes come together and annihilate each other to leave a single echo as in Figure 3a. (There is a considerable literature on optical focussing effects: their significance in radio-glaciology has been briefly discussed by Walford and Harper (1981).)

To return to the discussion of Figure $3 a$, we cannot assert that no focussing is produced by the shorter wavelength components of the bedrock profile. On the smaller scales, many echoes from the summits of ridges, from troughs, and from saddle points may contribute to the signal observed at a given point on the glacier surface, and what we then observe are echoes which vary in amplitude and pulse shape to a greater or lesser extent. Berry (1973) pointed out that we can treat this problem statistically and infer some of the statistical properties of the bed from the statistics of the echoes reflected from the bed.

The finest scale components of the rough glacier bed cannot be observed at all with our radio-echo sounder. Corrugations of the surface, which have a horizontal scale of less than $15 \mathrm{~m}(\lambda / 2)$ imprint merely evanescent perturbations on the reflected wave fronts and these are rapidly attenuated as the waves propagate upwards. The exception to this rule is scattering: observable echoes may be produced when radiation is scattered from discrete boulders or by water bodies at the bed, small compared with a wavelength. Such scattering cannot be treated within the framework of Berry's scalar theory of diffraction and we shall therefore discuss it separately.

In Figure $2 \mathrm{~d}$ are shown some A-scope records from Storglaciären. They have been selected to show, as clearly as possible, the range of variation of pulse shape exhibited by echoes from the bed. For this reason, we have chosen records which seem to show bed echoes relatively unobscured by echoes from internal targets. It is interesting to try to account for these variations in pulse shape. We shall consider the effects of gross curvature of the bed, of fine-scale roughness, of spatial variations of reflection coefficient, and of scattering. As we shall see, potentially useful information can be extracted from the variation of echo-pulse shape.

\section{THE EFFECT OF GROSS CURVATURE OF THE} GLACIER BED

Echo-pulse shape is weakly affected by large-scale curvature of the glacier bed. The effect can be calculated, rather precisely, using an expression for $g(r)$ for a smoothly curved hilltop derived by Berry (1972, appendix 2). Consider a smooth hilltop at a depth $h$, and let $R_{1}$ and $R_{2}$ be its principal radii of curvature at the point of minimum range. By convention the radii of curvature are taken as positive for a surface which is convex upward. Then, following Berry, we find

$$
\left.\begin{array}{lr}
g(r)=0, & (r<h) \\
g(r)=\frac{2 \pi Z}{h}\left[\frac{R_{1} R_{2}}{\left(h+R_{1}\right)\left(h+R_{2}\right)}\right]^{\frac{1}{2}}, \quad(r \geqslant h)
\end{array}\right\}
$$


where $Z$ is the amplitude reflection coefficient of the surface.

This expression is applicable to plane surfaces, for which $R_{1}$ and $R_{2}$ are infinite. It may also be used for surfaces which are concave as long as no focussing occurs. To illustrate the effect of bedrock curvature on echo-pulse shape, we computed $g(r)$ and the corresponding echo for three surfaces which were respectively, plane, spherically convex, and spherically concave. For the curved surfaces, we let $R_{1}=R_{2}=2 h$, and $-2 h$, respectively. (The condition for focussing is that either $R_{1}$ or $R_{2}$ be equal to $h$.) It was found that the most important effect of curvature is, not surprisingly, that the amplitude of the echo increases for the more concave surface. The pulse shape is hardly affected otherwise. Even the modest variation of pulse shape shown in Figure 2 cannot be accounted for by such curvature of the bedrock.

\section{ROUGHNESS OF THE GLACIER BED}

Echo-pulse shape may be a function of the roughness of the glacier bed. In this section, we suppose that the bed can be modelled in accordance with the assumptions underlying the scalar theory given by Berry (1973). We assume, in short, that it is a continuous, undulating surface of limited slope, without cracks or boulders. We also assume that it has a uniform reflection coefficient.

Berry's principal result was to show how the statistical quantities which we can measure from an ensemble of radio-echo records can be used to infer valuable statistical properties of the glacier bed. Humphrey (unpublished) has performed laboratory experiments which to some extent verify some of Berry's theoretical results but the theory has not been applied so far to the scattering of nearly monocycle pulses. Some of the approximations made by Berry in his analysis are somewhat poor in this regime and this will lead to systematic error. It is not likely to exceed the rather large random errors of measurement in the present application but it suggests a need for further work on the scattering of monocycle pulses by rough surfaces.

Berry assumed that the echoes may be validly regarded as samples drawn from a statistically stationary population of echoes. We have collected $169 \mathrm{~A}$-scope records at spatial intervals of $5 \mathrm{~m}$ over a rectangular array at site 1 (Fig. 1). This is in the ablation region of the glacier. The ice thickness varies slowly and the character of the echoes does not seem to change systematically in any obvious way within this data set, so we take them as independent samples from a population with rather well-defined statistics. Unfortunately, it is clear that there are echoes arising from the englacial water regime in this part of the glacier, and these corrupt some of our records significantly. We have therefore had to select 99 records on which the echo from the bed is not too greatly so affected.

Berry exemplified the significance of his results by showing how to distinguish, using statistical data from radio echoes, between three model surfaces. A surface which he denoted as surface $\alpha$ is a Gaussian rough surface which we may take as modelling the roughness of a glacier bed. His model surface $\gamma$ is a flat surface, the reflection coefficient of which varies as a function of position, in a Gaussian statistical way. This might be taken to model a smooth glacier bed with patches of water present. Berry's third model surface, B, consists of steps and is more suitable for modelling reflections from sea ice than from a glacier bed. To distinguish between the two relevant model surfaces $\alpha$ and $\gamma$, they must be studied at different frequencies. A monocycle pulse, of course, contains a wide band of frequencies, and so could in principle be used for this purpose.

In Berry's nomenclature, monocycle sounding at Storglaciăren is an example of diffraction near the long-wave limit. In this case, the wavelength is characteristically much longer than the height of the surface irregularities and the echo approximates to the shape of the transmitter pulse. The condition for the long-wave limit to apply is:

$$
\lambda>4 \pi S
$$

where $S$ is the root-mean-square deviation of the reflecting surface from a suitable smooth reference surface. Near the long-wave limit, the mean echo power as a function of delay time, $\langle P(t)\rangle$, consists of a major component, the coherent term, which is similar in form to $P_{\mathrm{T}}(t)$, the transmitted pulse power, together with a lesser, incoherent term which smooths and asymmetrically lengthens the mean echo power. Using our A-scope records from site 1 , we have plotted the mean echo power as a function of time in Figure 2e. Hence, we estimated the "coherency parameter", $\delta$, which Berry defined. It measures the mean contribution to the total returned echo power of the coherent part of the echo. We find that $\delta=0.7 \pm 0.2$. The r.m.s. height of irregularities, $S$, can now be found using Berry's formula 5.33:

$$
S=-\frac{\lambda}{4 \pi} \ln (6)
$$

Hence, at site 1 we find $S=0.8 \mathrm{~m}$ with the range of error from 0.3 to 2.0 .

Berry defined another important echo statistic, $T$, the echo half-length. It is a measure of the increase in echo length produced by the roughness of the bed. In our case, it is very small and difficult to measure: all we can really say is that it is probably less than $0.1 \mu \mathrm{s}$. Hence, using Berry's formula:

$$
L^{2}=2.77 h S^{2} / c T
$$

(Berry, 1973, equation 6.14), we estimate that the r.m.s. horizontal length $L$ of surface irregularities is not less than approximately $12 \mathrm{~m}$. This result is physically reasonable because, as we have said, components of the bedrock roughness with horizontal wavelengths less than $15 \mathrm{~m}$ produce evanescent waves which are strongly attenuated as they propagate upwards and are not generally observable.

In conclusion, the bed at Storglaciären appears to be rough on the finer scales measurable with our present lowfrequency echo-sounding equipment, as shown in region $B$ of Figure 4. On the larger scales, however, the glacier bed is extremely smooth.

\section{THE REFLECTIVITY OF THE GLACIER BED}

The pulse shape of a radio echo may depend on $Z$, the amplitude coefficient of reflection at the glacier bed. For waves reflected normally from the plane interface between two homogeneous dielectric media, $Z$ may be expressed in terms of the dielectric constants of the media, thus:

$$
Z=\frac{\sqrt{\epsilon_{i}}-\sqrt{\epsilon_{r}}}{\sqrt{\epsilon_{i}}+\sqrt{\epsilon_{r}}} .
$$

At radio frequencies $\epsilon_{\mathrm{i}}$, the dielectric constant of ice has the value of 3.18 and that of the bedrock $\epsilon_{\mathrm{r}}$ we shall take to be 2.7. $Z$ is therefore approximately 0.05 . But in Nature, the ice-rock boundary may not be a well-defined interface. In some places, it may be covered with a layer of drift or gravel and, if this is sufficiently thick to form a transition layer thicker than approximately $\lambda / 10$, then $Z$ will be reduced, for such a gradual transition between ice and rock presents no sharp discontinuity in wave impedance and very little reflection can occur. $Z$ may also be reduced if power is lost through scattering processes at the bed.

Water, having a high dielectric constant, can affect the reflection coefficient of the glacier bed even in quite small amounts. Ionic impurities in solution can be important if the conductivity of the water exceeds about $10^{-3} \mathrm{~S}$, as could occur for water in contact with the glacier bed. A few examples will be given to illustrate the role that water can play in determining the coefficient of reflection at the glacier bed.

Consider the bulk dielectric constant of water-saturated rock. It is a sensitive function of the geometry of the mixture of water and rock. In one extreme case, the water may be contained in cavities which are flattened perpendicular to 
the direction of the electric field. If the volume fraction occupied by water is $f_{\mathrm{v}}$, then the dielectric constant of this series arrangement of water and rock is:

$$
\epsilon_{\mathrm{S}}=\frac{\epsilon_{\mathrm{r}} \epsilon_{\mathrm{W}}}{\left(f_{\mathrm{v}} \epsilon_{\mathrm{r}}+\left(1-f_{\mathrm{v}}\right) \epsilon_{\mathrm{w}}\right)} .
$$

On the other hand, for an arrangement of water bodies long in a direction parallel to the electric field, the bulk dielectric constant is

$$
\epsilon_{\mathrm{p}}=\epsilon_{\mathrm{r}}\left(1-f_{\mathrm{v}}\right)+\epsilon_{\mathrm{w}} f_{\mathrm{v}} .
$$

For cases of intermediate geometry, an appropriate "mixtures formula" may be employed (van Beek, 1967). Using Equation (11), we find that as little as $0.6 \%$ of water suffices to increase the dielectric constant of rock to match that of ice and so reduced the reflection coefficient to zero. Using Equation (10), on the other hand, $16 \%$ of water would be necessary. A higher water concentration would, in either case, change the sign of the reflection coefficient, and this would invert the echo seen on an A-scope display.

Next we consider reflection at normal incidence, when there is a sheet of pure water of uniform thickness $D$ at the bed. The amplitude-reflection coefficient for continuous waves is given by the following expression (Born and Wolf, 1979):

$$
Z=\frac{Z_{\mathrm{iw}}+Z_{\mathrm{wr}} \exp (j 2 \phi)}{\left(1+Z_{\mathrm{iw}} Z_{\mathrm{ir}} \exp j 2 \phi\right)}
$$

where

$$
\phi=2 \pi D / \lambda \mathbf{w} .
$$

$\lambda_{w}$, the wavelength in the water layer, is approximately $5 \mathrm{~m}$ for our low-frequency apparatus. $Z_{\text {iw }} \approx 0.668$ is the amplitude coefficient of reflection at an ice-water interface and $Z_{w r} \approx-0.691$ is that at a water-rock interface. We find, from Equation (12), that if $D \ll 0.6 \mathrm{~m}$, then

$$
Z \sim 0.05+j 17 D / \lambda_{w}
$$

$Z$ is complex and dependent on the radio frequency. It follows that in this case the pulse shape of the echo differs from that of the incident pulse $F(t)$. The most extreme example arises when $Z \sim j 17 D / \lambda_{w}$, which is approximately the case if $0.6 \mathrm{~m} n D n 0.01 \mathrm{~m}$. In this case, it can be shown by Fourier analysis that the echo pulse is approximately the same as the first derivative with respect to time of the incident pulse. Thus, an incident pulse with the wave form of Figure 2a would produce an echo like Figure 2b. The radio echoes collected in the field vary in pulse shape as indicated in Figure 2d. On the average, however, the pulse shape of the echoes seems more like $F(t)$ than its derivative $F^{\prime}(t)$. If this is the correct interpretation, it suggests that no substantial water layer was present at the bed when the echoes were recorded in September 1980. This is interesting in view of measurements showing that during the summer months the glacier height increased over the lower part of Storglaciären by as much as $0.20 \mathrm{~m}$ (Hooke and others, 1983[b]).

Having considered how a rather uniform sheet of water at the bed could influence the pulse shape of a radio echo, we now ask whether the statistical fluctuations of the pulse shape, which we illustrated in Figure 2d, could be accounted for in terms of spatial variations in the thickness of this water layer? Diffraction by such spatial variations provides an alternative model to the one we considered in the previous section, which dealt with diffraction by roughness at the bed. Let us assume for the moment that the principal effect of water is to produce a spatial variation of the reflection coefficient. The model is now of Berry's surface $\gamma$, and accordingly we substitute the values of the coherence parameter and the echo half-length into Berry's (1973) formulae 5.34 and 6.15. Hence, we find that the horizontal scale of the variations of reflection coefficient is of the order of $15 \mathrm{~m}$ and that

$$
\langle Z\rangle^{2} \sim 0.7\left\langle Z^{2}\right\rangle \text {. }
$$

To interpret this result, consider a simple model in which a fraction $f_{S}$ of the area of the bed has a constant reflection coefficient $Z_{1}$ and the rest has $Z=0$. The different regions are typically $15 \mathrm{~m}$ across. On this mode $\langle Z\rangle=f_{\mathrm{s}} Z_{1}$ and $\left\langle Z^{2}\right\rangle=f_{\mathrm{s}} Z_{1}^{2}$, and so it follows, from Equation (14), that $f_{\mathrm{S}} \sim 0.7$. Physically, this model might correspond to a glacier bed, $70 \%$ of which is covered with a rather substantial layer of water, $0.10 \mathrm{~m}$ thick say. Although in theory one can discriminate between the model surfaces $\alpha$ and $\gamma$ on the basis of the frequency dependence of echo statistics, in the present case the incoherent contribution to the echo is too small for us to do so.

\section{SCATTERING AT THE GLACIER BED}

Scattering here, means the diffraction of radio waves by discrete bodies, the dimensions of which are small compared with $\lambda / 2$. Such bodies, distributed over an otherwise smooth glacier bed, would modify the nearly specular nature of the reflection, just as sub-microscopic dust or scratches degrade an optical flat. Water bodies in the form of sufficiently small pools, or streams at the bed, could produce rather strong back-scattered echoes because $\epsilon_{w}$ is relatively large. Boulders or ice-filled cavities within the bedrock would scatter more weakly. Scattering cannot be described in terms of the scalar-wave diffraction theory of Berry because, by definition, the necessary condition that the surface of the target be a smoothly varying, monotonic surface is violated. Instead, one needs to use a suitable vector-wave theory such as the theory of Rayleigh scattering. We should expect to find that, if the bed was a smooth surface bearing a small number of scatterers, the echo would be a superposition of a specularly reflected coherent pulse and many scattered wavelets which would combine in random phase to produce a long incoherent echo tail. We cannot tell whether such a thing occurs at Storglaciären, because there is such strong scattering from englacial targets that any such long tail is obscured. Therefore, we pursue the matter no further.

\section{SUMMARY}

We now wish to summarize the discussion of radio echoes returned from the glacier bed. In theory, an echo from a smooth ice-rock interface has the form of $F(t)$. If the interface is covered by a few centimetres of water, however, the echo form is $F^{\prime}(t)$. In theory, spatial fading of the echoes could be due to roughness of the ice-rock interface, to spatial variations in reflection coefficient associated perhaps with water, or to scattering by discrete small bodies. In principal, one might distinguish between these three cases by spectral analysis. In practice the echoes from Storglaciären depart only slightly from the form of the incident wave and we cannot clearly decide which, if any, of the spatial fading mechanisms is dominant. Nevertheless, some useful approximate maximum values have been given for the roughness of the bed and for the statistics of a spatially variable reflection coefficient. However, scattering near the bed cannot be clearly distinguished from englacial scattering which we consider next.

\section{RADIO ECHOES FROM ENGLACIAL TARGETS}

In this section we discuss the measurement and analysis of radio echoes from targets within the glacier. We have recorded such echoes, systematically, on three occasions at Storglaciăren. First, in September 1981, we obtained photographic A-scope records from an array of 169 stations at site 1 (Fig. 1) using the low frequency model B instrument. In September 1984 we obtained both A-scope and I-scope records from nine parallel traverses at site 2 using the model S instrument. Also we obtained A-scope records, for three different polarization states, at 20 stations along the line of site 3 , using the $65 \mathrm{MHz}$ instrument. The data from 
all of these experiments have been analysed in order to obtain statistical information about the echo power, the fading patterns, and the polarization produced by englacial targets.

Let us consider radio-echo power as a function of delay time. We calculated the mean power, $\langle P(t)\rangle$, from measurements made on 54 independent A-scope records of low-frequency echoes at sites 1 and 2 . The absolute value of the echo power is not known very reliably because we have imperfect knowledge of the behaviour of radio antennae lying on the surface of a glacier. The relative accuracy of $\langle P(t)\rangle$ as a function of time is better known: it is limited by the small number of records and by the restricted interval in the time domain within which reliable measurements can be made from the photographs. No echoes are observed at $t<0.3 \mu \mathrm{s}$ but this could be attributable to the fact that the direct wave from the transmitter to the receiver tends to obscure echoes at such short delay times, as can be seen in Figure $3 \mathrm{~b}$. The echo power decreases as the delay time increases and for $t>2.0 \mu \mathrm{s}$ it becomes difficult to make reliable measurements from photographic records. The origin of time is not known precisely for the low-frequency instruments because of the way in which the oscilloscope is triggered from the signal which travels directly from the transmitting to the receiving antenna. We can calculate the origin of time only roughly from a knowledge of the antennae separation; it is better to find the origin experimentally, by making suitable measurements of echoes returned from a known target, such as a straight, conducting cable lying at a known range on the surface of the glacier. Alternatively, we can avoid the difficulty altogether, by plotting $\langle P(t)\rangle^{n}$ as a function of time. We find that a straight line is obtained in the interval $0.4 \mu \mathrm{s}<t<2.0 \mu \mathrm{s}$ if $n=-0.5 \pm 0.1$ (Fig. 5).

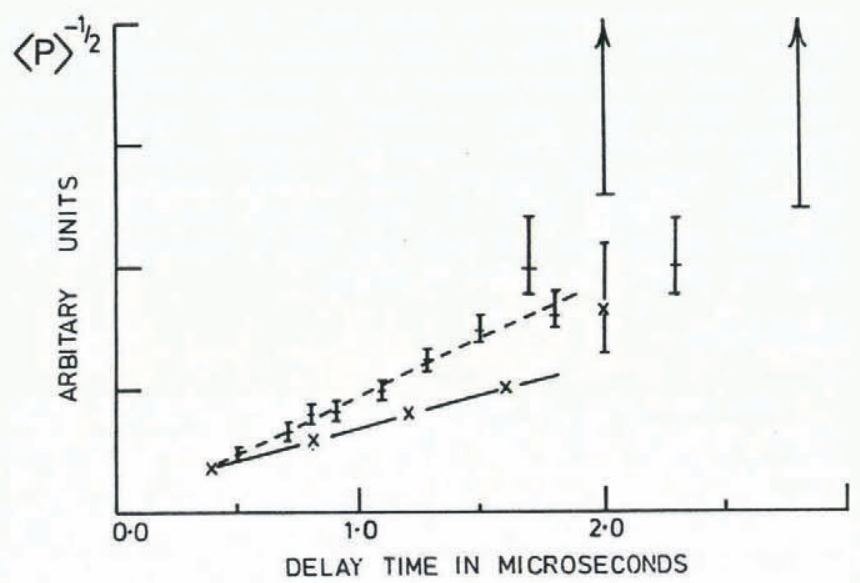

Fig. 5. The reciprocal of mean radio-echo amplitude (measured without regard to sign), as a function of time delay.

$\times$ average of 26 echo records from site 1 , using system B.

+ average of 28 echo records from site 2 and elsewhere, using system $S$.

We may account for this inverse-square dependence of the mean echo power upon delay time by, for example, a model in which small, identical targets are distributed at random in the glacier. If the mean number per unit volume is $N$ then the mean number per unit interval of range, $r$, is $2 \pi N r^{2}$. The echo power from an individual target is

$$
\frac{P_{\mathrm{T}}(t-2 r / c) \sigma A G}{\left(4 \pi r^{2}\right)^{2}}
$$

where $P_{\mathrm{T}}(t)$ represents the power transmitted as a function of time, $\sigma$ is the back-scattering cross-section for an individual target, $A$ is the effective area of the receiving antenna, and $G$ the power gain of the transmitting antenna. If the targets are distributed at random, it is appropriate to sum the power contributions of each target in order to calculate $\langle P(t)\rangle$. We find

$$
\langle P(t)\rangle=\int_{0}^{\infty} \frac{P_{\mathrm{T}}(t-2 r / c) \sigma A G 2 \pi N r^{2} \mathrm{~d} r}{\left(4 \pi r^{2}\right)^{2}} \approx \frac{E \sigma N A G}{4 \pi c t^{2}}
$$

where

$$
E=\int_{0}^{\infty} P_{\mathrm{T}}(t) \mathrm{d} t
$$

is the total energy transmitted. We see that if targets are distributed statistically at random through the glacier the mean radio-echo power should vary as $t^{-2}$ as is observed in practice. For comparison, to take an extreme case, if the targets were scattered on the glacier surface, the mean echo power would vary as $t^{-3}$.

To proceed further, it would be very interesting to form an estimate, however approximate, of the value of $N \sigma$. To do this it is necessary to estimate the efficiency of the radio antennae, in order to calculate the transmitted energy $E$ from a knowledge of the electrical energy supplied by the transmitter circuit to the transmitting antenna. We also need the antenna efficiency in order to calculate the radio-echo power from measurements of the power delivered to the receiver from the receiving antenna. Following Kanda (1980), we have taken the value of 0.08 for the efficiency of each antenna. We assumed a figure of 1.6 for the antenna gain. These values are appropriate for antennae in free space and take no account of the fact that our antennae are working close to the air-ice interface. As a rough check on the validity of these assumptions, we have calculated the signal radiated directly from one antenna to another parallel to it a few wavelengths away. The calculations agree, to within an order of magnitude, with a field measurement of the signal. We have therefore carried through the approximate calculation for the radio-echo targets and found that $N \sigma \sim 3 \times 10^{-4} \mathrm{~m}^{-1}$, for the lowfrequency echoes. Because we cannot take into account properly the effects of the glacier on the antennae, this value of $N \sigma$ should be regarded as no more than a rough, order-of-magnitude result.

Now let us consider the case of the $65 \mathrm{MHz}$ echoes. We have again calculated the mean power as a function of delay time for the experiments when the antennae were parallel to each other. There is no measurable power at $t<0.3 \mu \mathrm{s}$ in either of these experiments. This cannot now be attributed to masking effects because the direct signal is much smaller at $65 \mathrm{MHz}$ than it was for the low-frequency system. We believe that the lack of echoes at $t<0.3 \mu \mathrm{s}$ indicates an absence of targets at depths of less than $30-40 \mathrm{~m}$. (A similar effect was first noted during traverses on Storglaciăren in April 1984, using an $80 \mathrm{MHz}$ geophysical impulse sounder (personal communication from P. Ulriksen).) It has been attributed to a surface layer of ice at temperatures significantly below $0{ }^{\circ} \mathrm{C}$.

At $65 \mathrm{MHz}$ the mean echo power could be fitted quite well to a function of the form

$$
\langle P(t)\rangle \propto t^{-2} \exp -t / \tau
$$

where $\tau$ is a constant which has the value approximately $1 \mu \mathrm{s}$. The exponential term may indicate either a systematic decrease in $N \sigma$ as a function of depth or it may be attributable to attenuation due to absorption or scattering of the radio signal as it propagates through the glacier. (It may be noted that $\frac{1}{2} c \tau$ gives an attenuation length which is of order $100 \mathrm{~m}$.)

We used the $65 \mathrm{MHz}$ echo records obtained with the antennae parallel to each other to estimate a value of $N \sigma$. We find a value $N \sigma=0.05 \mathrm{~m}^{-1}$. Despite the limited accuracy of the estimates, we believe that this is significantly greater than $3 \times 10^{-4} \mathrm{~m}^{-1}$, the value obtained at $6 \mathrm{MHz}$ with the low-frequency instrument.

Next, we consider the statistical description of the fading of echoes from englacial targets. Figure $3 \mathrm{~b}$ is a typical example from the set of nine low-frequency I-scope 
records obtained at site 2, well away from unwanted instrumentation cables. At delay times greater than approximately $2.5 \mu \mathrm{s}$, we see several rather continuous echo traces which probably arise by reflection from different parts of the glacier bed. (Björnsson's (1981) map shows the glacier to be relatively deep and narrow near this site.) The bands which cross the record at $t=0-0.3 \mu \mathrm{s}$ are produced by the direct signal from the transmitter. The irregular pattern of echoes between 0.3 and $2.5 \mu \mathrm{s}$ is produced by scattering from englacial targets. Within this interval the fading pattern seems to be statistically uniform, apart from the decrease in amplitude with increasing delay time. It is statistically symmetrical with respect to reversal, either of the time axis or the space axis.

In principle, one could describe such spatial fading in terms of a mean-power spatial-density function or equivalently, a two-dimensional autocorrelation function. We hope to do this in the future but, for the present, it is sufficient to estimate the temporal and spatial fading rates by counting the number of zero crossings per unit interval in each case. We find, for these low-frequency records, a mean of approximately 13 or 14 zero crossings per microsecond in the time domain and 3 or 4 per $100 \mathrm{~m}$ traverse. It is interesting to note that the temporal fading rate is significantly greater than the value, $12 \mu \mathrm{s}^{-1}$, which one would expect, given that the centre frequency of the transmitted pulse is $6 \mathrm{MHz}$. This effect indicates that the scattering process may be frequently dependent. In this context it is worth remarking that Smith and Evans (1972) discussed radio echoes from temperate glaciers in terms of two mode processes, both of which are dispersive. They are, respectively, reflections from a parallel-sided water layer and Rayleigh scattering by small spheres. As we remarked earlier, if the pulse $F(t)$ is incident on a thin layer, the echo has the form $F^{\prime}(t)$, as a result of dispersion on reflection. In the case of the Rayleigh scattering, dispersion is even more pronounced. The power scattered from a component wave of angular frequency $\boldsymbol{w}$ is proportional to $\omega^{4}$ and the phase of the scattered wave is independent of frequency (Born and Wolf, 1979). From this it can be shown, by Fourier analysis, that a pulse scattered from a single sphere has the form $F^{\prime \prime}(t)$. We have calculated $F^{\prime}(t)$ and $F^{\prime \prime}(t)$ by a differencing method, from the pulse $F(t)$ shown in Figure 2a. The resulting wave forms are plotted in Figure $2 b$ and $c$.

An echoing wave field produced by the superposition of pulses of form $F(t)$ would be characterized by a mean zero-crossing frequency of $\sim 12 \mu \mathrm{s}^{-1}$. For pulses $F^{\prime}(t)$ and $F^{\prime \prime}(t)$, the corresponding frequencies are $\sim 15 \mu \mathrm{s}^{-1}$ and $\sim 18 \mu \mathrm{s}^{-1}$, respectively. One may compare these values with the experimental result, $13-14 \mu \mathrm{s}^{-1}$, in order to obtain an indication of the extent to which dispersive processes occur in the field.

There is one further observation to make, which appears to be significant. At some places, rather coherent trains of wave cycles occur, which seem to persist for periods two or three times longer than the duration of the transmitter signal. They persist spatially for 10 to $20 \mathrm{~m}$. Jacobel and Raymond (1984) observed similar returns using a $16 \mathrm{MHz}$ instrument and attributed them to multiple scattering from water-filled cavities. The effect, however, is not well understood and merits further study.

Next, we discuss spatial fading; in general, the spatial fading of echoes from englacial targets is expected to depend statistically on the frequency spectrum of the scattered waves and on the angular distribution of their propagation vectors. The latter, in turn, depends on the polar diagrams of the antennae and of the reflecting targets. Following Robin and others (1969), we find that the ratio of the temporal and spatial fading rates is theoretically $c / 2 \sin \beta$ where $c$ is the velocity of propagation in ice and $B$ is a useful measure of the angular distribution of propagation vectors. From our low-frequency experimental data at site 2 , we find $B \sim 13^{\circ}$.

We can obtain further insight into the nature of radioecho fading by carrying out, in the computer, experiments which simulate the scattering of scalar pulses by englacial targets. Figure 6 shows the fading pattern generated by one such experiment. The synthetic pattern is statistically quite similar to our field records and has approximately the same

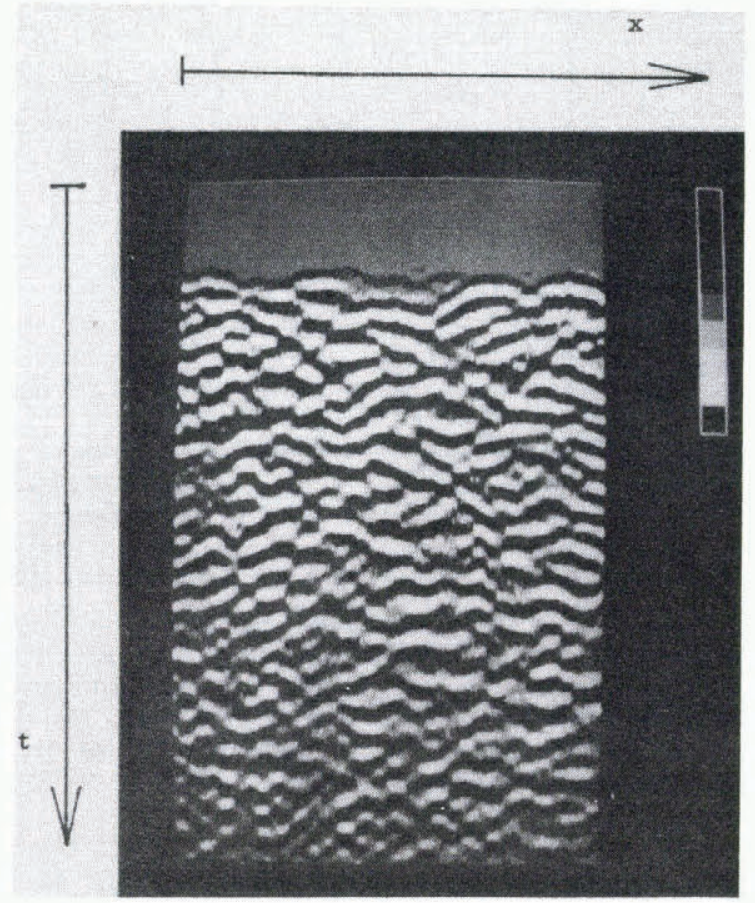

Fig. 6. A synthetic I-scope record produced by a computer model. The glacier contained $2.7 \times 10^{-3}$ isotropic targets per $\mathrm{m}^{3}$, distributed at random in three dimensions below 30-40 $\mathrm{m}$ depth. The computer modelled the transmitted wave form and the polar diagram of the antennae realistically. The longitudinal and vertical scales correspond approximately to those shown in Figure 3.

value of B. It does not reproduce the occasional, anomalously long, coherent wave trains mentioned above. It also differs in exhibiting several well-defined and nearvertical lines across which the phase of the echoes changes abruptly. These lines are probably associated with the presence of wave-front dislocations in the echoing wave field (Nye and Berry, 1974; Humphrey, unpublished). Dislocations are generic features of any wave field. That we do not see them so clearly in the field records is probably due to the relatively low contrast and poor resolution of the latter.

In order that a synthetic record should resemble a field record, it is necessary, as far as possible, to use a realistic pulsed wave form and antennae polar diagrams. There must also be a sufficient number of echoing targets, otherwise the synthetic records are dominated by hyperbolae. To produce Figure 6 , for example, $2.7 \times 10^{-3}$ isotropic targets per $\mathrm{m}^{3}$ were distributed at random in three dimensions, below a depth of $\sim 30 \mathrm{~m}$.

Finally, in this section, we discuss an experiment carried out using the $65 \mathrm{MHz}$ instrument to study the polarization of radio echoes. Measurements were made at 20 stations along the line of site 3 (Fig. 1). There was no evidence of any systematic variation of the echoes along this line and so spatial averages of the echo power were calculated as a function of echo delay time $t$. We obtained three such functions $\left\langle P_{11}(t)\right\rangle$ for the case when both antennae were parallel to site $3,\left\langle P_{22}(t)\right\rangle$ for the case when both antennae were perpendicular to site 3 , and $\left\langle P_{12}(t)\right\rangle$ when the transmitting antenna was parallel and the receiving antenna was perpendicular to site 3 .

In no case were substantial echoes received from less than $30 \mathrm{~m}$ depth. Beyond this, the ratios $\left\langle P_{12}(t)\right\rangle \mid\left\langle P_{11}(t)\right\rangle$ and $\left\langle P_{22}(t)\right\rangle /\left\langle P_{11}(t)\right\rangle$ do not vary sytematically with $t$. Accordingly we calculated mean values for these two ratios, which were $0.58 \pm 0.05$ and $0.85 \pm 0.05$, respectively. We represent this experimental result by the point labelled $F$ in Figure 7.

To assess the significance of this result, it it important to take account of the effects of antennae polar diagram as well as possible polarizing effects produced by the target. In order to illustrate the importance of antennae polar 


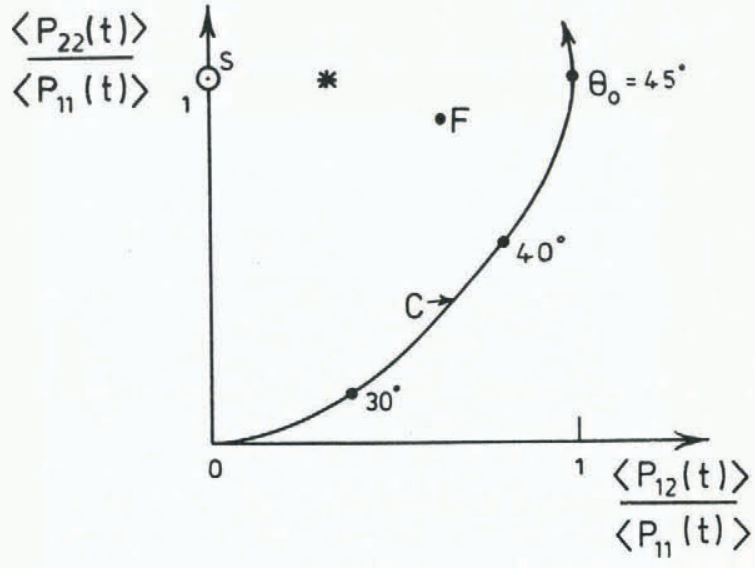

Fig. 7. Mapping of the polarization characteristics of radio echoes. $\left\langle P_{11}(t)\right\rangle$ is the spatially averaged echo power with the antennae parallel to site 3. The suffix 2 indicates an antenna perpendicular to this line. $\mathrm{F}$ represents the field data polarization and $\mathrm{S}, \mathrm{N}, \mathrm{F}$, and $\mathrm{C}$ refer to various models discussed in the text.

diagram, let us assume for the moment that, in the horizontal plane, the amplitude polar diagram of each dipole antenna is a sinusoidal function of azimuthal angle. For an isotropic scatterer lying on the snow surface at a bearing $\theta_{0}$ with respect to the axis of site 3 , it follows that $\left.\left\langle P_{12}(t)\right\rangle\right\rangle$ $\left\langle P_{11}(t)\right\rangle=\tan ^{-2} \theta_{0}$ and $\left\langle P_{22}(t)\right\rangle\left\langle\left\langle P_{11}(t)\right\rangle=\tan ^{-4} \theta_{0}\right.$. Thus, returns from this scatterer map on to the line $C$ in Figure 7 at a point determined by $\theta_{0}$.

To illustrate the polarizing effects of various targets, let us now suppose that the polar diagram of each antenna has circular symmetry about the downward direction. The radio echoes are now quite insensitive to the polar-diagram effect and map at a point on Figure 7 determined by target symmetry alone. For example, echoes from spherical targets would map at point $s$, with coordinates $(0,1)$. Echoes from needle-like targets, orientated at random in the horizontal plane, would map at $\mathrm{N}$, with coordinates $(0.33,1.0)$. Echoes from needles lying parallel to the azimuth would map on the line c, just as did echoes from spheres in the example of the previous paragraph. Thus an ambiguity can arise which can be resolved only by moving the radio-echo sounder about on the snow surface.

The experimental result represented by point $\mathbf{F}$ in Figure 7 could at first sight be, to some extent, the result of the polar-diagram effect. But there was no evidence of any systematic variations of echo range or polarization properties as the echo-sounder was moved along the line of site 5. It seems probable, therefore, that the radio-echo targets beneath site 3 do exhibit a preferred alignment at approximately $42^{\circ}$ to the line of the site.

To summarize this section, we find that $\langle P(t)\rangle$ can be accounted for in terms of a random spatial distribution of targets below about $30 \mathrm{~m}$ depth in the ice. Estimates of $\mathrm{NO}$ and of temporal fading rates both suggest that the scattering process is somewhat frequency dependent. Polarization studies show that, at site 2 and at $65 \mathrm{MHz}$, targets appear to be non-isotropic and have some preferred orientation in azimuth.

\section{THE PHYSICAL NATURE OF THE ENGLACIAL TARGETS}

Following Smith and Evans (1972), we suppose that it is the presence of liquid water which makes the essential differences between the weak but often highly stratified echoes from within Antarctic polar ice, and the much stronger, but unstratified echoes from Storglaciăren Certainly, Storglaciarren is a very wet glacier in summer. Schytt (1981) found that up to $2.5 \mathrm{~m}$ of ice may be melted from the surface on average during a good summer. Seasonal variation in surface velocity and elevation have been interpreted in terms of seasonal variations in the water regime (Brzozowski and Hooke, 1981; Hooke and others, 1983[b]). However, measurements of temperature in the upper few tens of metres do suggest that there is a surface layer of ice at temperatures below the melting point which may extend down to $30-40 \mathrm{~m}$ (Schytt, 1968; Hooke and others, 1983[a]). This may account for the absence of radio echoes from shallow depths.

We expect that the water distribution within Storglaciăren has structure on various scales. On a large scale, we observe water in moulins, crevasses, and surface streams, and we note fossil remains of such features exposed at the surface in the ablation zone. There may also be water distributed on intermediate scales right down to water films, filaments, and droplets of microscopic dimensions. Statistical variations of such smaller-scale features may cause radio echoes. However, the most effective targets are probably water-filled and, to a lesser extent, air-filled cavities, the dimensions of which are at least comparable with the radar wavelength. We believe our radio-echo experiments give some insight into the distribution of such features.

\section{CONCLUSION}

We have attempted to analyse radio echoes from the bed and from within Storglaciären and we have, we believe, obtained useful, if approximate, statistical measures of such parameters as bedrock roughness, and the distribution, strength, and orientation of englacial targets. General difficulties in such work include the problem of knowing the antennae properties when they are used near to the glacier surface, and of knowing to what extent it is valid to treat the echoes as samples drawn from a statistically, stationary population. It is clearly necessary, in the future, to obtain much more data in order to improve the statistical significance of our results. We hope to do this and to extend our studies to include echo-sounding at various different frequencies. We shall use aperture synthesis and study the slow changes of echoes which occur as a function of time.

\section{ACKNOWLEDGEMENTS}

This research was partly supported by U.K. N.E.R.C. grant GR3/4883. We acknowledge the inspiration and help provided by the late Professor V. Schytt, whose death earlier this year was such a great loss to glaciology. We also thank Professor J.F. Nye, Professor R. Hooke, Dr R. Jacobel, and Dr M.F.L. Harper for help and encouragement at various stages.

\section{REFERENCES}

Beek, L.K.H. van. 1967. Dielectric behaviour of heterogeneous systems. (In Birks, J.B., ed. Progress in dielectrics. Vol. 7. London, Heywood Books, p. 69-114.)

Berry, M.V. 1972. On deducing the form of surfaces from their diffracted echoes. Journal of Physics. A: General Physics, Vol. 5, No. 2, p. 272-91.

Berry, M.V. 1973. The statistical properties of echoes diffracted from rough surfaces. Philosophical Transactions of the Royal Society of London, Ser. A, Vol. 272, No. 1237 , p. 611-54.

Born, M., and Wolf, E. 1979. Principles of optics. Oxford, Pergamon Press.

Björnsson, H. 1981. Radio-echo sounding maps of Storglaciăren, Isfallsglaciăren and Rabots glaciăr, northern Sweden. Meddelanden fran Naturgeografiska Institutionen vid Stockholms Universitet, Nr. A 124, p. 123-25.

Brzozowski, J., and Hooke, R.L. 1981. Seasonal variations in surface velocity of the lower part of Storglaciären, Kebnekaise, Sweden. Meddelanden frän Naturgeografiska Institutionen vid Stockholms Universitet, Nr. A 125, p. 233-40.

Hooke, R.L., and others. 1983[a]. Near-surface temperatures near and below the equilibrium line on polar and subpolar glaciers, by R.L. Hooke, J.E. Gould, and J. Brzozowski. Zeitschrift für Gletscherkunde und Glazialgeologie, Bd. 19, Ht. 1, p. 1-25. 
Hooke, R.L., and others. 1983[b]. Seasonal variations in surface velocity, Storglaciăren, Sweden, by R.L. Hooke, J. Brzozowski, and C. Bronge. Meddelanden frän Naturgeografiska Institutionen vid Stockholms Universitet, Nr. A 150 , p. 263-77.

Humphrey, V.F. Unpublished. Experimental observations of wavefront dislocations in pulsed wavefields. [Ph.D. thesis, University of Bristol, 1980.]

Jacobel, R., and Raymond, C. 1984. Radio echo-sounding studies of englacial water movement in Variegated Glacier, Alaska. Journal of Glaciology, Vol. 30, No. 104, p. 22-29.

Kanda, M. 1980. Transients in a resistively loaded antenna compared with those in a conical horn and a T.E.M. horn. IEEE Transactions on Antennas and Propagation, Vol. AP-28, No. 1, p. 132-36.

Nye, J.F., and Berry, M.V. 1974. Dislocations in wave trains. Proceedings of the Royal Society of London, Ser. A, Vol. 330 , p. $165-90$.

Robin, G. de Q., and others. 1969. Interpretation of radio echo sounding on polar ice sheets, by G. de Q. Robin, S. Evans, and J.T. Bailey. Philosophical Transactions of the Royal Society of London, Ser. A, Vol. 265, No. 1166, p. 437-505.
Schytt, V. 1968. Notes on glaciological activities in Kebnekaise, Sweden, during 1966 and 1967. Geografiska Annaler, Vol. 50A, No. 2, p. 111-20.

Schytt, V. 1981. The net mass balance of Storglaciären, Kebnekaise, Sweden, related to the height of the equilibrium line and to the height of the $500 \mathrm{mb}$ surface. Meddelanden fran Naturgeografiska Institutionen vid Stockholms Universitet, Nr. A 123, p. 219-23.

Shen, L.C., and King, R.W.P. 1965. The cylindrical antenna with nonreflecting resistive loading. IEEE Transactions on Antennas and Propagation, Vol. Ap-13, p. 998.

Smith, B.M.E., and Evans, S. 1972. Radio echo sounding: absorption and scattering by water inclusion and ice lenses. Journal of Glaciology, Vol. 11, No. 61, p 133-46.

Walford, M.E.R., and Harper, M.F.L. 1981. The detailed study of glacier beds using radio-echo techniques. Geophysical Journal of the Royal Astronomical Society, No. 67 , p. 487-514.

Wu, T.T., and King, R.W.P. 1965. The cylindrical antenna with nonreflecting resistive loading. IEEE Transactions on Antennas and Propagation, Vol. Ap-13, p. 369-73. 